\title{
High-fat diet decreases energy expenditure and expression of genes controlling lipid metabolism, mitochondrial function and skeletal system development in the adipose tissue, along with increased expression of extracellular matrix remodelling- and inflammation-related genes
}

\author{
Myung-Sook Choi ${ }^{1,2} \dagger$, Young-Je Kim ${ }^{1} \dagger$, Eun-Young Kwon ${ }^{2}$, Jae Young Ryoo ${ }^{3}$, Sang Ryong Kim $^{3}$ \\ and Un Ju Jung ${ }^{2 *}$ \\ ${ }^{1}$ Department of Food Science and Nutrition, Kyungpook National University, 1370 San-Kyuk Dong Puk-Ku, \\ Daegu 702-701, Republic of Korea \\ ${ }^{2}$ Center for Food and Nutritional Genomics Research, Kyungpook National University, 1370 San-Kyuk Dong Puk-Ku, \\ Daegu 702-701, Republic of Korea \\ ${ }^{3}$ School of Life Sciences, BK21 Plus KNU Creative BioResearch Group, Kyungpook National University, \\ 1370 San-Kyuk Dong Puk-Ku, Daegu 702-701, Republic of Korea
}

(Submitted 13 August 2014 - Final revision received 13 November 2014 - Accepted 22 December 2014 - First published online 6 March 2015)

\section{Abstract}

The aim of the present study was to identify the genes differentially expressed in the visceral adipose tissue in a well-characterised mouse model of high-fat diet (HFD)-induced obesity. Male C57BL/6J mice ( $n$ 20) were fed either HFD (189\% of energy from fat) or low-fat diet (LFD, $42 \%$ of energy from fat) for 16 weeks. HFD-fed mice exhibited obesity, insulin resistance, dyslipidaemia and adipose collagen accumulation, along with higher levels of plasma leptin, resistin and plasminogen activator inhibitor type 1, although there were no significant differences in plasma cytokine levels. Energy intake was similar in the two diet groups owing to lower food intake in the HFD group; however, energy expenditure was also lower in the HFD group than in the LFD group. Microarray analysis revealed that genes related to lipolysis, fatty acid metabolism, mitochondrial energy transduction, oxidation-reduction, insulin sensitivity and skeletal system development were down-regulated in HFD-fed mice, and genes associated with extracellular matrix (ECM) components, ECM remodelling and inflammation were up-regulated. The top ten up- or down-regulated genes include Acsm3, mt-Nd6, Fam13a, Cyp2e1, Rgs1 and Gpnmb, whose roles in the deterioration of obesity-associated adipose tissue are poorly understood. In conclusion, the genes identified here provide new therapeutic opportunities for prevention and treatment of diet-induced obesity.

Key words: High-fat diet: Energy expenditure: Global gene expression in adipose tissue: Inflammation

Obesity is one of the major underlying causes of the metabolic syndrome, including insulin resistance, type 2 diabetes and dyslipidaemia. Although the exact mechanisms by which obesity induces or worsens the metabolic risk factors are still unknown, excess adiposity, particularly visceral fat accumulation, is associated with insulin resistance and abnormal glucose and lipid metabolisms ${ }^{(1)}$. In addition, chronic lowgrade inflammation in the adipose tissue contributes to the pathogenesis of insulin resistance and metabolic syndrome ${ }^{(1)}$.

Diet composition plays an important role in the development of obesity and its associated metabolic diseases ${ }^{(2)}$.
Dietary fat is the most energy-dense macronutrient and causes less satiety than carbohydrate or protein ${ }^{(2)}$. Prolonged ingestion of high-fat diet (HFD) has been found to induce hyperphagia, body-weight gain and fat deposition and increase the levels of circulating glucose, insulin and TAG in rats ${ }^{(3-5)}$. However, results have been inconsistent, with some studies reporting that HFD did not cause hyperphagia, hypertriglycerolaemia, hyperglycaemia or hyperinsulinaemia in mice ${ }^{(6,7)}$.

Carbohydrates, especially simple sugars, also seem to be associated with obesity and metabolic syndrome. Longterm ingestion of a sugar-rich diet increases fat mass without

\footnotetext{
Abbreviations: ECM, encoding extracellular matrix; GPCR, G-protein-coupled receptors; HFD, high-fat diet; HOMA-IR, homeostatic index of insulin resistance; LFD, low-fat diet; RGS, regulators of G protein signalling; WAT, white adipose tissue.
}

*Corresponding author: Dr U. J. Jung, fax +82 53958 1230, email jungunju@naver.com

†These authors contributed equally to this work. 
a concomitant increase in energy intake ${ }^{(8)}$ and promotes insulin resistance in rats $^{(9)}$. Furthermore, a low-fat, highcarbohydrate diet had deleterious effects on cardiovascular health in obese adults compared with a high-fat, lowcarbohydrate diet ${ }^{(10)}$. In contrast, another study has shown that a high-fat, low-carbohydrate diet decreases serum TAG levels and increases serum HDL-cholesterol levels in obese subjects, without concomitant changes in body weight or composition, in comparison with a low-fat, highcarbohydrate diet ${ }^{(11)}$. These results show that the role of diet in obesity varies with diet composition, animal species and experimental protocol.

Recently, nutritional genomics studies have analysed the responses of tissues to different diets and nutrients in order to provide a insight into the molecular events underlying diet-induced obesity ${ }^{(12)}$. However, obesity-related metabolic and molecular changes in response to HFD (containing low carbohydrate) $v$. low-fat diet (LFD; containing high carbohydrate) are not yet fully understood. Moreover, the HFD used in most animal studies of diet-induced obesity contains an extremely high fat content (approximately 60\% of total energy) ${ }^{(11)}$, which does not mimic the moderate fat content of Western human diets (approximately $40-45 \%$ of total energy).

Therefore, the primary aim of the present study was to compare the effects of long-term ingestion of HFD (189\% of energy from fat) and LFD (which provides more carbohydrate, namely maize starch and sugar) on food intake, energy expenditure, body weight and adiposity, as well as plasma glucose, insulin, lipid and adipocytokine profiles, in C57BL/6J mice. The secondary aim of the present study was to obtain a further insight into the molecular mechanisms underlying the development of diet-induced obesity and its related metabolic abnormalities in response to HFD and to identify differentially expressed genes in the epididymal white adipose tissue (WAT) of HFD-fed mice using microarray analysis.

\section{Experimental methods}

\section{Feeding protocol, energy expenditure and} blood biomarkers

Male C57BL/6J mice (4-week old) were purchased from Jackson Laboratories and individually housed at room temperature on a $12 \mathrm{~h}$ light $-12 \mathrm{~h}$ dark cycle. After 1 week of acclimation, they were fed a LFD (D12450B, $16.17 \mathrm{~kJ} / \mathrm{g}$; Research Diets) or a HFD (D12451, 19.866 kJ/g; Research Diets) ad libitum for 16 weeks. The LFD contains $42 \%$ of energy from fat $(25 \cdot 2 \%$ of energy from soyabean oil and $16.8 \%$ of energy from lard), $294 \%$ of energy from carbohydrate $(130 \cdot 2 \%$ of energy from maize starch, $16.8 \%$ of energy from maltodextrin and $147 \%$ of energy from sucrose) and $84 \%$ of energy from protein, whereas the HFD contains $189 \%$ of energy from fat $(25 \cdot 2 \%$ of energy from soyabean oil and $163.8 \%$ of energy from lard), $147 \%$ of energy from carbohydrate $(29 \cdot 4 \%$ of energy from maize starch, $42 \%$ of energy from maltodextrin and $75.6 \%$ of energy from sucrose) and $84 \%$ of energy from protein. Food intake of each mouse was measured daily throughout the study by subtracting the remaining food from the amount of food given to the mice, and daily food intake was calculated from the averaged food intake throughout the study. Energy expenditure for $24 \mathrm{~h}$ was measured at 14 weeks of feeding on the experimental diets using an indirect calorimeter (Oxylet; Panlab). Mice were killed as described previously ${ }^{(6)}$, and epididymal WAT, perirenal WAT, retroperitoneal WAT, mesenteric WAT and subcutaneous WAT were promptly removed, rinsed with physiological saline and weighed after blood collection. Among them, the epididymal WAT, which is widely used for the metabolic study due to its anatomically distinct feature, relative abundance and metabolic sensitivity ${ }^{(13-18)}$, was immediately fixed in $10 \%$ buffered formalin for morphological examination and was frozen in liquid $\mathrm{N}_{2}$ and stored at $-70^{\circ} \mathrm{C}$ until RNA analysis. Plasma concentrations of NEFA, phospholipid (Wako Chemicals), TAG, total cholesterol and HDL-cholesterol (Asan Pharmaceutical Co., Ltd) were determined using commercially available kits.

Plasma adipocytokine and insulin levels were determined using a multiplex detection kit (Bio-Rad) and analysed using a Luminex 200 Labmap system. Fasting blood glucose concentration was measured using a glucose analyser (Glucocard; Arkray), and the homeostatic index of insulin resistance (HOMA-IR) was calculated as (fasting glucose $(\mathrm{mmol} / \mathrm{l}) \times$ fasting insulin $(\mathrm{pmol} / \mathrm{l}) / 135)$. All the experimental procedures were approved by the Kyungpook National University Ethics Committee (Approval no. KNU-2011-49).

\section{Morphology of the epididymal white adipose tissue}

The epididymal WAT was fixed in $10 \%$ buffered formalin. Fixed tissues were embedded in paraffin, and $4 \mu \mathrm{m}$ sections were prepared and stained with haematoxylin and eosin and Masson's trichrome. The stained areas were viewed using an optical microscope (Nikon) with a magnification of $200 \times$, and epididymal adipocyte size and fibrotic area from the Masson's trichrome staining were measured by computer analysis using the Leica Application Suite (version 2.8.1; Leica Microsystems).

\section{RNA isolation, microarray analysis and real-time quantitative $P C R$}

Total RNA was extracted from the epididymal WAT using TRIzol reagent (Invitrogen Life Technologies). For quality control, RNA purity and integrity were assessed using an Agilent 2100 Bioanalyzer (Agilent Technologies). Three pooled RNA sample sets were constructed to represent the LFD and HFD groups, as described previously ${ }^{(19)}$.

For microarray analysis, total RNA was amplified and purified using an Illumina RNA amplification kit (Ambion ${ }^{\circledR}$ ) and quantified using an ND-1000 spectrophotometer (NanoDrop). Biotinylated CRNA $(750 \mathrm{ng}$ ) was hybridised to MouseWG-6 v2 0 Expression BeadChips (Illumina, Inc.) at $58^{\circ} \mathrm{C}$ for $16-18 \mathrm{~h}$. Array signal detection was carried out using Amersham Cy3streptavidin (GE Healthcare Bio-Sciences). BeadChips were scanned using an Illumina BeadArray Reader, and raw data 
were extracted using the Illumina BeadStudio software. Probe signal intensities were quantile-normalised and log-transformed. Limma was used to determine significantly differentially expressed genes based on a false discovery rate less than $5 \%$, a Benjamin and Hochberg-adjusted $P$ value $<0.05$ and a $\log _{2}$ fold change greater than $1^{(19)}$. The DAVID Functional Annotation Tool was used to identify the enriched biological themes and cluster-redundant annotation terms. These microarray data were deposited in Gene Expression Omnibus database (accession no. GSE63198).

To validate microarray data, several differentially expressed genes (Rgs1, Mmp2, Ccl2, Tlr2, Tlr4 and Irs2) were measured independently by real-time quantitative PCR using the same pooled RNA samples that were hybridised to BeadChips. Total RNA $(1 \mu \mathrm{g})$ was reverse-transcribed into complementary DNA using a QuantiTect reverse transcription kit (Qiagen), and real-time quantitative PCR was carried out on a CFX96 real-time system (Bio-Rad) using a SYBR Green PCR kit (Qiagen). Values were normalised to glyceraldehyde-3phosphate dehydrogenase levels, and relative gene expression was calculated by the $2^{-\Delta \Delta \mathrm{Ct}}$ method.

For definition of gene abbreviations, see online Supplementary Table S1.

(a)

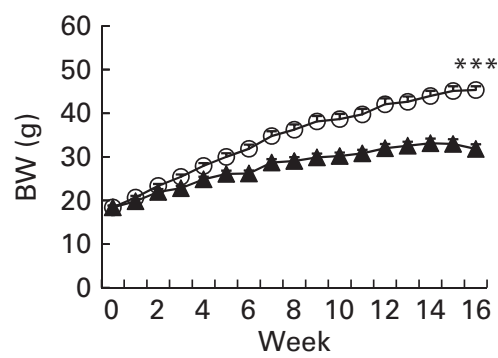

(b)

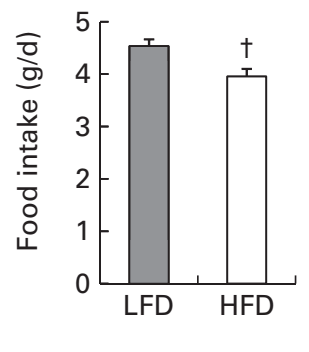

\section{Statistical analyses}

All data are presented as means with their standard errors. Statistical analyses were performed using SPSS software. Changes in body weight were analysed by repeated-measures ANOVA, and other data were analysed by Student's $t$ test or Wilcoxon $t$ test. Results were considered statistically significant when $P<0 \cdot 05$.

\section{Results}

High-fat diet decreased food intake but increased body weight and fat mass by decreasing energy expenditure

HFD-fed mice exhibited a significantly greater body weight than LFD-fed mice, although the average daily food intake throughout the study was markedly lower in HFD-fed mice and the average daily energy intake did not differ between the two groups (Fig. 1(a)-(c)). Interestingly, indirect calorimetry revealed that HFD-fed mice had lower energy expenditure than LFD-fed mice (Fig. 1(d)). As expected, the subcutaneous WAT and the visceral WAT (including epididymal, perirenal, retroperitoneal and mesenteric WAT) weights, adipocyte size and adipose collagen accumulation were higher in HFD-fed mice (Fig. 1(e) and (f)).

(c)

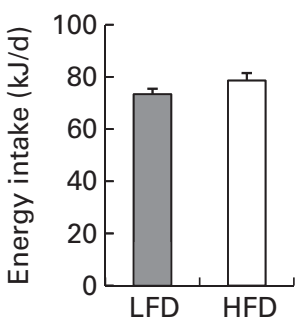

(d)

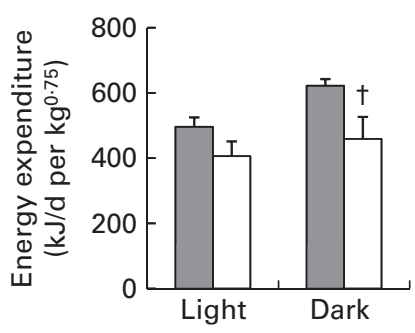

(e)

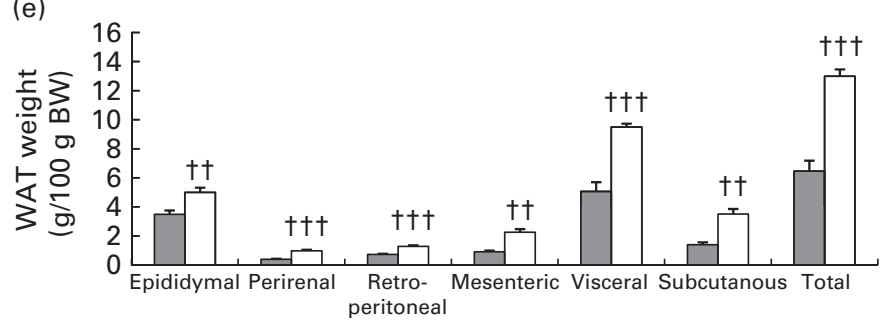

(f)

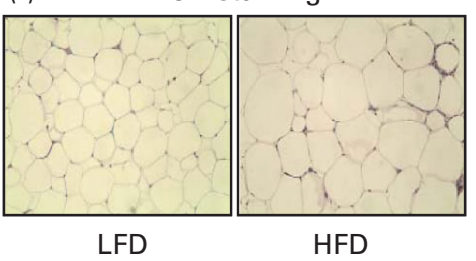

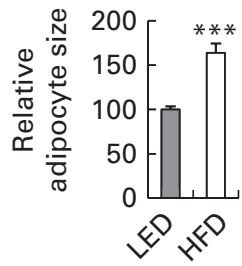
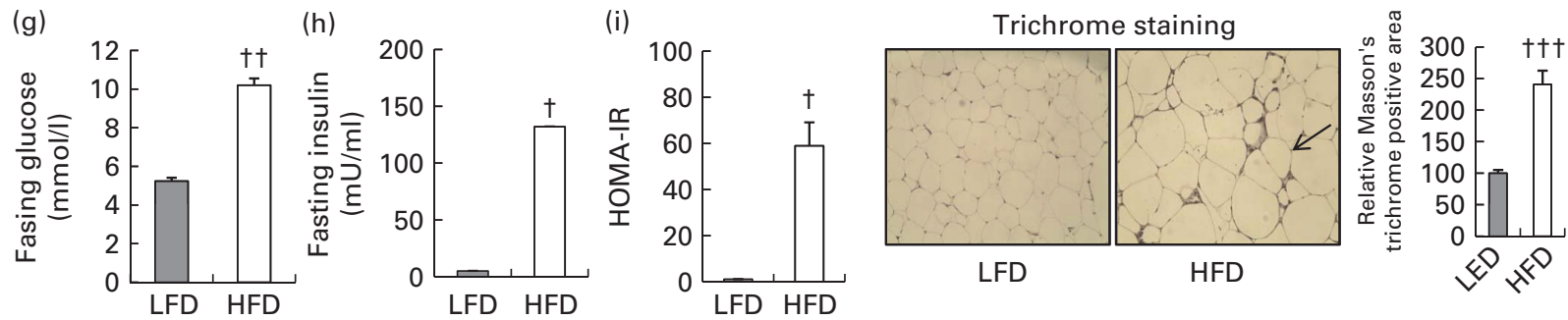

Fig. 1. Metabolic and morphological phenotype of high-fat diet (HFD, $\odot, \square)$-fed mice. Values are means with their standard errors represented by vertical bars $(n 10)$. (a) ${ }^{* * *}$ Mean value was significantly different from that of low-fat diet (LFD, $\left.-\rightarrow\right)$ group $(P<0.001$; repeated-measures ANOVA). (b-i) Mean value was significantly different from that of low-fat diet (LFD, $\square$ ) group: $\dagger P<0.05, \dagger \dagger P<0.01, \dagger+\uparrow P<0.001$ (Student's $t$ test). (f) Representative photographs of adipocytes in the epididymal white adipose tissue (WAT) of mice at $\times 200$ magnification (left panel) and quantitative analysis (right panel). The WAT section stained with Masson's trichrome showed significant deposition of collagens, primarily collagen I and III (blue stain indicated with arrowheads), in HFD-fed mice. BW, body weight; H\&E, haematoxylin and eosin; HOMA-IR, homeostatic index of insulin resistance. (A colour version of this figure can be found online at http://www.journals.cambridge.org/bjn) 
Table 1. Plasma adipocytokine and lipid levels

(Mean values with their standard errors; $n$ 10)

\begin{tabular}{|c|c|c|c|c|}
\hline & \multicolumn{2}{|c|}{ LFD } & \multicolumn{2}{|c|}{ HFD } \\
\hline & Mean & SE & Mean & SE \\
\hline \multicolumn{5}{|l|}{ Adipocytokines } \\
\hline Leptin (ng/ml) & 2.83 & 0.79 & $57 \cdot 13^{\star \star \star}$ & $6 \cdot 11$ \\
\hline Resistin (ng/ml) & $208 \cdot 74$ & 32.04 & $575 \cdot 91^{\star * *}$ & 23.79 \\
\hline PAl-1 (ng/ml) & 1.26 & 0.74 & $2 \cdot 42^{\star \star}$ & 0.32 \\
\hline Adiponectin $(\mu \mathrm{g} / \mathrm{ml})$ & 11.38 & 1.27 & 13.43 & 0.66 \\
\hline MCP-1 (pg/ml) & 248.44 & $26 \cdot 69$ & 234.71 & $30 \cdot 12$ \\
\hline TNF- $\alpha(p g / m l)$ & 2016.99 & 267.50 & 1633.68 & 275.39 \\
\hline IL-6 (pg/ml) & 23.36 & 3.05 & 22.74 & 3.66 \\
\hline $\mathrm{IL}-10(\mathrm{pg} / \mathrm{ml})$ & $920 \cdot 36$ & 93.69 & 850.00 & 85.90 \\
\hline \multicolumn{5}{|l|}{ Lipids } \\
\hline NEFA $(\mathrm{mmol} / \mathrm{l})$ & 0.90 & 0.07 & 0.91 & 0.07 \\
\hline TAG $(\mathrm{mmol} / \mathrm{l})$ & 0.81 & 0.08 & 1.04 & 0.07 \\
\hline Phospholipid (mmol/l) & 0.92 & 0.15 & $2 \cdot 45^{\star}$ & 0.15 \\
\hline Total cholesterol $(\mathrm{mmol} / \mathrm{l})$ & $2 \cdot 61$ & 0.28 & $4 \cdot 34^{\star *}$ & 0.32 \\
\hline HDL-cholesterol $(\mathrm{mmol} / \mathrm{l})$ & 1.35 & 0.19 & $1.98^{*}$ & 0.18 \\
\hline Atherosclerotic index & 1.26 & 0.33 & 1.26 & 0.19 \\
\hline
\end{tabular}

LFD, low-fat diet; HFD, high-fat diet; PAI-1, plasminogen activator inhibitor type 1; MCP, monocyte chemotactic protein.

Mean values were significantly different from those of the LFD group: ${ }^{*} P<0.05$, ${ }^{* *} P<0.01,{ }^{* * *} P<0.001$.

High-fat diet induced hyperglycaemia, hyperinsulinaemia and insulin resistance

Fasting blood glucose level was significantly increased in HFD-fed mice (Fig. 1(g)). HFD also resulted in significant increases in plasma insulin level and HOMA-IR compared with LFD (Fig. 1(h) and (i)).

High-fat diet increased plasma leptin, resistin and plasminogen activator inhibitor type 1 levels but did not alter plasma adiponectin and cytokine levels

Plasma leptin, resistin and plasminogen activator inhibitor type 1 levels were significantly higher in HFD-fed mice than in LFD-fed mice (Table 1). However, there was no significant difference in plasma adiponectin level between the two groups. The levels of plasma cytokines (monocyte chemotactic protein 1 , TNF- $\alpha$, IL-6 and IL-10) were also not significantly altered by HFD.

\section{High-fat diet increased plasma phospholipid, total cholesterol and HDL-cholesterol levels but did not alter plasma NEFA and TAG levels}

No significant differences in plasma NEFA or TAG levels were observed between the HFD and LFD groups (Table 1). However, plasma phospholipid level was significantly higher in HFD-fed mice. HFD also increased plasma total cholesterol level, as well as HDL-cholesterol level, resulting in no significant difference in atherogenic index between the two groups.

\section{Gene expression profiles in the epididymal white adipose tissue}

To determine the changes in global gene expression profiles of the epididymal WAT in HFD-induced obesity, we identified differentially expressed genes in HFD-fed mice compared with LFD-fed mice using microarray analysis. Of the 45000 analysed expression probes, 1270 were different between the two groups. Among these 1270 HFD-responsive genes, 657 were up-regulated and 397 were down-regulated. The top ten differentially expressed genes are listed in Table 2. Functional annotation clustering using DAVID revealed that the majority of genes up-regulated by HFD were related to immune and inflammatory responses (Table 3; online Supplementary Table S2). These included genes encoding chemokines, receptors of chemokines and cytokines, tolllike receptors, C-type lectin receptors, Fc receptors and surface markers of immune cells (Table 4). Moreover, HFD induced up-regulation of genes encoding extracellular matrix (ECM) components such as collagen (Col1a1, Col3a1, Col5a2, Col6a2 and Col6a3), glycosaminoglycan and proteoglycan (Sdcbp and Lum), adhesive glycoproteins (Mfap5, Gpnmb and Fn1) and integrin (Itgad, Itgam and Itgax), as well as genes encoding proteins involved in ECM remodelling and regulation, such as cathepsins (Ctsa, Ctsh, Ctsk, Ctsl, Ctss and $C t s z$ ), a disintegrin and metalloprotease (ADAM) domain (Adam8, Adam12 and Adam17), matrix metalloproteinases (MMP: Mmp2, Mmp3, Mmp12 and Mmp13), tissue inhibitors of metalloproteinases (Timp1) and other fibrosis-related genes ( $T g f b 1)$. Interestingly, the expression of two $A D A M$ and $M M P$ genes, Adam 7 and $M m p 9$, was down-regulated in obesity.

The genes that were down-regulated in response to HFD were enriched in gene ontology categories related to oxidation-reduction, fatty acid metabolism, insulin response and skeletal system development (Table 3; online Supplementary Table S2). Down-regulated genes related to oxidation-reduction included genes controlling antioxidant defence (Gpx3), detoxification (Aldb1a1, Aldb6a1, Cyp2d9, Cyp2d22, Cyp2e1, Cyp2f2, Gsta3 and Gsta4) and mitochondrial energy transduction pathways such as the TCA cycle (Por) and oxidative phosphorylation (Ndufb4 and Ndufb9) (Table 4). HFD decreased the expression of genes involved in lipolysis, thermogenesis (Adrb3) and fatty acid uptake, transport (Fabp4, Cd36 and Slc27a2), elongation (ElovlO), activation and oxidation (Acsm3, Acacb, Acot4, Acadsb, $H a d h$ and $F a a b$ ). Consistent with increased insulin resistance,

Table 2. Top ten differentially expressed genes in the epididymal white adipose tissue of high-fat diet-fed mice

\begin{tabular}{ll}
\hline & Differentially expressed genes* \\
\hline $\begin{array}{l}\text { Up-regulated genes } \\
\text { Down-regulated genes }\end{array}$ & Rgs1, Mmp12, Gpnmb, Trem2, Tph2, Saa3, Ubd, Atf3, Itgad, Cd68 \\
\hline $\begin{array}{l}\text { * Differentially expressed genes were determined using Limma in R/Bioconductor, based on } P<0.05, \text { false discovery rate }<5 \% \text { and log } 2 \text { fold change } \\
>1 . \text { For definition of gene abbreviations, see online Supplementary Table S1. }\end{array}$
\end{tabular}


Table 3. Functional annotation clusters of up-regulated and down-regulated genes in the epididymal white adipose tissue of high-fat diet-fed C57BL/6J mice*

\begin{tabular}{llrr}
\hline ES & Function & Count & $P$ \\
\hline Up-regulated genes & & & \\
13.67 & Immune response & 63 & $7.00 \times 10^{-21}$ \\
& Inflammatory response & 39 & $4.10 \times 10^{-16}$ \\
& Response to wounding & 47 & $3.70 \times 10^{-15}$ \\
& Defence response & 49 & $9.30 \times 10^{-13}$ \\
6.21 & Cell activation & 38 & $1.20 \times 10^{-14}$ \\
& Cell proliferation & 20 & $9.80 \times 10^{-04}$ \\
5.94 & Chemotaxis/taxis & 22 & $1.30 \times 10^{-10}$ \\
5.09 & Positive regulation of immune response & 23 & $6.90 \times 10^{-10}$ \\
4.64 & Positive regulation of response to stimulus & 24 & $7.90 \times 10^{-08}$ \\
4.02 & Regulation of cytokine production & 24 & $3.40 \times 10^{-10}$ \\
3.95 & Phagocytosis & 12 & $5.40 \times 10^{-07}$ \\
3.14 & Cell division & 25 & $5.70 \times 10^{-05}$ \\
& Cell cycle & 41 & $9.00 \times 10^{-05}$ \\
2.68 & Positive regulation of immune system process & 33 & $3.20 \times 10^{-13}$ \\
& Negative regulation of immune system process & 12 & $4.80 \times 10^{-05}$ \\
Down-regulated genes & & & \\
4.71 & Oxidation-reduction & 36 & $1.90 \times 10^{-07}$ \\
4.44 & Fatty acid metabolism & 19 & $1.40 \times 10^{-07}$ \\
2.42 & Response to insulin stimulus & 9 & $5.40 \times 10^{-04}$ \\
2.08 & Skeletal system development and morphogenesis & 17 & $8.80 \times 10^{-06}$ \\
& Pattern specification process & 16 & $1.60 \times 10^{-04}$ \\
\hline
\end{tabular}

ES, enrichment score.

* Functional annotation terms were clustered according to biological processes.

insulin sensitivity-related genes, such as those encoding insulin receptor substrate 2 (Irs2) and GLUT 3 (Slc2a3), were down-regulated by HFD feeding. Along with $M m p 9$, multiple genes controlling skeletal system development and pattern specification (Bmp4, Hoxa5, Hoxa 7, Hoxb5, Hoxc6, $\operatorname{Irx} 3$ and $\operatorname{Igf2}$ ) were also down-regulated in HFD-fed mice. The expression of many genes encoding G-protein-coupled receptors (GPCR: Gpr64, Gpr120, Gpr109a, Gpr156 and Opn3) was decreased in response to HFD, whereas mRNA expression of regulators of $G$ protein signalling (RGS: Rgs1 and Rgs10) was increased. Several genes identified as differentially expressed based on microarray analysis were independently validated by real-time quantitative PCR (Fig. 2).

\section{Discussion}

It is generally accepted that HFD induces hyperphagia, and that increased energy intake is the major mechanism by which HFD causes obesity ${ }^{(2,3)}$. In the present study, however, despite lower food intake and similar energy intake, mice fed HFD (189\% of energy from fat) for 16 weeks gained more body weight and body fat than mice fed LFD ( $42 \%$ of energy from fat), which provided more energy as carbohydrate (sugar and maize starch). This is consistent with a previous finding that body weight and adiposity, but not energy intake, increased in HFD-fed C57BL/6J mice compared with LFD-fed mice during the first 17 weeks of feeding, although energy intake was significantly higher in HFDfed mice after 17 weeks owing to a reduction in leptin sensitivity ${ }^{(20)}$. Body weight is normally maintained by a balance between energy intake and energy expenditure. When energy intake exceeds energy expenditure, excess energy is stored as TAG in the adipose tissue, resulting in overweight or obesity. Because energy expenditure during dark hours was significantly lower in HFD-fed mice than in LFD-fed mice, the increase in body weight and fat mass in HFD-fed mice may be due to a positive energy balance resulting from a decrease in energy expenditure relative to energy intake.

Induction of energy expenditure based on fatty acid oxidation in WAT may reduce adiposity ${ }^{(21)}$. Mitochondrial biogenesis and thermogenesis are decreased in WAT of obese individuals and rodents ${ }^{(21-23)}$, while induction of mitochondria and activation of fatty acid oxidation have been observed in WAT under conditions promoting loss of adiposity ${ }^{(21)}$. Kusminski and Scherer ${ }^{(24)}$ suggested that in the obese state, mitochondria in WAT cannot cope with increasing demands for fatty acid oxidation, resulting in incomplete $\beta$-oxidation. In the present study, HFD down-regulated the expression of genes involved in fatty acid catabolism and oxidation, as well as genes controlling the mitochondrial energy transduction pathways, including the TCA cycle and oxidative phosphorylation, in the epididymal WAT. In other studies, expression of Faah (a catabolic gene for bioactive fatty acid amides) in adipose tissue was negatively correlated with visceral fat mass in human subjects ${ }^{(25)}$, and mice lacking Faab had increased body weight and fat mass with decreased energy expenditure $^{(26)}$. Thus, down-regulation of genes regulating fatty acid catabolism, fatty acid oxidation and energy metabolism in epididymal WAT may be partly responsible for the decrease in energy expenditure induced by HFD. Our findings also suggest that the deleterious effects of HFD on adipose tissue mitochondria are associated with decreased expression of genes involved in antioxidant defence and detoxification. An altered redox state is associated with the accumulation of products of incomplete $\beta$-oxidation and increased mitochondrial reactive oxygen species formation, leading to a 
Table 4. Fold changes of selected genes influenced by high-fat diet*

\begin{tabular}{|c|c|c|c|c|c|}
\hline \multicolumn{3}{|c|}{ Up-regulated genes } & \multicolumn{3}{|c|}{ Down-regulated genes } \\
\hline Symbol & Fold change & $P$ & Symbol & Fold change & $P$ \\
\hline Chemokines and their receptors & & & Oxidation-reduction & & \\
\hline $\mathrm{Ccl} 2$ & 3.08 & $3.62 \times 10^{-04}$ & Gpx3 & $-2 \cdot 36$ & 0.002 \\
\hline $\mathrm{Ccl} 3$ & $2 \cdot 62$ & $8.21 \times 10^{-05}$ & Aldh1a1 & $-2 \cdot 19$ & 0.001 \\
\hline $\mathrm{Ccl} 4$ & 3.37 & $1.66 \times 10^{-04}$ & Aldh6a1 & -2.31 & 0.001 \\
\hline Ccl6 & 3.74 & $5.73 \times 10^{-05}$ & Сур2d9 & -3.45 & 0.011 \\
\hline Ccl7 & $3 \cdot 10$ & $5.93 \times 10^{-04}$ & Сур2d22 & -2.93 & 0.001 \\
\hline Ccl9 & $5 \cdot 34$ & $2.64 \times 10^{-06}$ & Cyp2e1 & -4.69 & 0.006 \\
\hline Cxcl2 & 2.04 & $4.86 \times 10^{-05}$ & Сур2f2 & -4.42 & $3.28 \times 10^{-04}$ \\
\hline Cxcl12 & $2 \cdot 10$ & $9.45 \times 10^{-05}$ & Gsta3 & -2.32 & $3.48 \times 10^{-05}$ \\
\hline Cxcl14 & 2.03 & 0.003197 & Gsta4 & -3.90 & $3.02 \times 10^{-05}$ \\
\hline Cxcl16 & $2 \cdot 17$ & $4.72 \times 10^{-05}$ & Por & $-2 \cdot 19$ & $2.13 \times 10^{-04}$ \\
\hline Ccr5 & $6 \cdot 00$ & $1.28 \times 10^{-05}$ & Ndufb4 & -2.02 & $3.24 \times 10^{-04}$ \\
\hline Cxcr4 & 2.94 & $1.19 \times 10^{-04}$ & Ndufb9 & $-2 \cdot 36$ & $3.89 \times 10^{-04}$ \\
\hline Cytokine and trigger receptors & & & Lipolysis and fatty acid metabolism & & \\
\hline II7r & $15 \cdot 01$ & $1.03 \times 10^{-06}$ & Adrb3 & $-3 \cdot 78$ & 0.005127 \\
\hline Trem2 & 31.19 & $7.63 \times 10^{-07}$ & Fabp4 & -9.01 & $7.63 \times 10^{-07}$ \\
\hline Toll-like receptors & & & Cd36 & -3.58 & $2.93 \times 10^{-06}$ \\
\hline TIr1 & $4 \cdot 28$ & $3.36 \times 10^{-05}$ & Slc27a2 & $-2 \cdot 42$ & 0.032965 \\
\hline TIr2 & 2.53 & $9.58 \times 10^{-05}$ & Elovl6 & $-2 \cdot 61$ & 0.046248 \\
\hline TIr6 & 2.44 & $4.27 \times 10^{-04}$ & Acsm3 & $-12 \cdot 76$ & $2.34 \times 10^{-04}$ \\
\hline TIr7 & $2 \cdot 76$ & $5.96 \times 10^{-04}$ & Faah & -2.04 & 0.015 \\
\hline Tlr8 & 5.96 & $3.86 \times 10^{-05}$ & $A c a c b$ & $-4 \cdot 12$ & 0.001 \\
\hline TIr13 & $14 \cdot 84$ & $1.27 \times 10^{-06}$ & Acot4 & $-3 \cdot 61$ & 0.002 \\
\hline TIr7 & $2 \cdot 76$ & $5.96 \times 10^{-04}$ & Acadsb & -2.08 & 0.002 \\
\hline C-type lectin receptors & & & Hadh & $-2 \cdot 73$ & $8.72 \times 10^{-06}$ \\
\hline Clec4a1 & $2 \cdot 52$ & $7.61 \times 10^{-05}$ & Insulin sensitivity & & \\
\hline Сlec4a3 & $3 \cdot 81$ & $1.20 \times 10^{-05}$ & Irs2 & -2.78 & 0.005 \\
\hline Clec4b1 & 4.96 & $1.40 \times 10^{-05}$ & Slc2a3 & -3.05 & $1.40 \times 10^{-05}$ \\
\hline Clec4d & $9 \cdot 98$ & $6.35 \times 10^{-05}$ & $\begin{array}{l}\text { Skeletal system development and pattern } \\
\text { specification process }\end{array}$ & & \\
\hline Clec7a & $5 \cdot 84$ & $9.06 \times 10^{-06}$ & Bmp4 & -2.54 & 0.001 \\
\hline Clecsf12 & 8.02 & $8.64 \times 10^{-06}$ & Hoxa5 & $-2 \cdot 21$ & $5.21 \times 10^{-05}$ \\
\hline Fc receptors & & & Hoxa7 & -2.55 & $7.07 \times 10^{-05}$ \\
\hline Fcgr1 & 3.44 & $2.28 \times 10^{-04}$ & Hoxb5 & -2.31 & 0.001 \\
\hline Fcgr3 & $6 \cdot 26$ & $5.97 \times 10^{-06}$ & Hoxc6 & -2.09 & $4.92 \times 10^{-04}$ \\
\hline Fcgr4 & 4.74 & $1.75 \times 10^{-04}$ & Irx3 & -2.33 & 0.004 \\
\hline Fcer1a & 2.46 & $4.63 \times 10^{-04}$ & $\operatorname{lgf2}$ & $-2 \cdot 60$ & $2.26 \times 10^{-04}$ \\
\hline Fcer1g & 5.51 & $7.18 \times 10^{-06}$ & Mmp9 & $-2 \cdot 18$ & $2.38 \times 10^{-05}$ \\
\hline Surface markers of immune cells & & & G-protein-coupled receptors and others & & \\
\hline Cd9 & 4.06 & $1.56 \times 10^{-05}$ & Gpr64 & -2.85 & 0.02 \\
\hline Cd14 & $2 \cdot 26$ & $6.79 \times 10^{-04}$ & Gpr120 & -2.83 & $4.81 \times 10^{-04}$ \\
\hline Cd44 & 8.35 & $2.49 \times 10^{-06}$ & Gpr109a & -2.57 & 0.002 \\
\hline Cd53 & 4.75 & $2.21 \times 10^{-05}$ & Gpr156 & $-2 \cdot 19$ & $1.13 \times 10^{-04}$ \\
\hline Cd63 & $2 \cdot 12$ & $5.20 \times 10^{-05}$ & Opn3 & -2.29 & 0.001 \\
\hline Cd68 & $15 \cdot 72$ & $7.85 \times 10^{-07}$ & Adam7 & -2.220 & 0.04 \\
\hline Cd72 & 9.73 & $6.30 \times 10^{-06}$ & & & \\
\hline Cd83 & $4 \cdot 14$ & $1.90 \times 10^{-04}$ & & & \\
\hline Cd84 & $12 \cdot 41$ & $4.04 \times 10^{-06}$ & & & \\
\hline Cd93 & 2.04 & $1.08 \times 10^{-04}$ & & & \\
\hline Cd180 & 5.92 & $1.24 \times 10^{-05}$ & & & \\
\hline Cd248 & 2.09 & 0.001 & & & \\
\hline \multicolumn{6}{|l|}{ ECM components } \\
\hline Col1a1 & 4.71 & $5.66 \times 10^{-05}$ & & & \\
\hline Col3a1 & $2 \cdot 64$ & $8.51 \times 10^{-05}$ & & & \\
\hline Col5a2 & $2 \cdot 65$ & $5.99 \times 10^{-05}$ & & & \\
\hline Col6a2 & 2.42 & 0.001966 & & & \\
\hline Col6a3 & $2 \cdot 39$ & $7.15 \times 10^{-05}$ & & & \\
\hline Sdcbp & 2.95 & $4.74 \times 10^{-05}$ & & & \\
\hline Lum & 4.80 & $1.23 \times 10^{-05}$ & & & \\
\hline Mfap5 & $2 \cdot 71$ & 0.001 & & & \\
\hline Gpnmb & 31.53 & $8.61 \times 10^{-07}$ & & & \\
\hline Fn1 & 2.58 & 0.001236 & & & \\
\hline Itgad & $17 \cdot 29$ & $7.85 \times 10^{-07}$ & & & \\
\hline Itgam & 2.00 & $8.15 \times 10^{-04}$ & & & \\
\hline Itgax & $3 \cdot 37$ & $7.06 \times 10^{-05}$ & & & \\
\hline \multicolumn{6}{|l|}{ ECM remodelling and regulation } \\
\hline Ctsa & $4 \cdot 16$ & $7.96 \times 10^{-07}$ & & & \\
\hline
\end{tabular}


Table 4. Continued

\begin{tabular}{|c|c|c|c|c|c|}
\hline \multicolumn{3}{|c|}{ Up-regulated genes } & \multicolumn{3}{|c|}{ Down-regulated genes } \\
\hline Symbol & Fold change & $P$ & Symbol & Fold change & $P$ \\
\hline Ctsh & 2.52 & $4.81 \times 10^{-05}$ & & & \\
\hline Ctsk & $13 \cdot 18$ & $7.85 \times 10^{-07}$ & & & \\
\hline Ctsl & $4 \cdot 14$ & $6.47 \times 10^{-05}$ & & & \\
\hline Ctss & 4.93 & $5.89 \times 10^{-05}$ & & & \\
\hline Ctsz & 3.23 & $8.17 \times 10^{-05}$ & & & \\
\hline Adam8 & $6 \cdot 87$ & $1.33 \times 10^{-05}$ & & & \\
\hline Adam 12 & $2 \cdot 12$ & $2.89 \times 10^{-04}$ & & & \\
\hline Adam17 & 2.37 & $4.86 \times 10^{-05}$ & & & \\
\hline Mmp2 & 4.02 & $2.16 \times 10^{-05}$ & & & \\
\hline Mmp3 & $4 \cdot 17$ & $2.91 \times 10^{-05}$ & & & \\
\hline Mmp12 & 31.86 & $1.97 \times 10^{-05}$ & & & \\
\hline Mmp13 & 3.44 & $1.95 \times 10^{-05}$ & & & \\
\hline Timp1 & 7.90 & $4.74 \times 10^{-05}$ & & & \\
\hline Tgfb1 & 2.64 & $1.20 \times 10^{-04}$ & & & \\
\hline \multicolumn{6}{|c|}{ Regulator of $G$ protein signalling } \\
\hline Rgs1 & 33.98 & $5.75 \times 10^{-07}$ & & & \\
\hline Rgs 10 & 3.58 & $3.03 \times 10^{-05}$ & & & \\
\hline
\end{tabular}

* Differentially expressed genes were determined using Limma in R/Bioconductor, based on $P<0.05$, false discovery rate $<5 \%$ and log ${ }_{2}$ fold change $>1$. For definition of gene abbreviations, see online Supplementary Table S1.

deterioration of insulin sensitivity ${ }^{(27)}$. Mitochondrial reactive oxygen species produced by redox imbalance may promote adipocyte differentiation ${ }^{(28)}$. In adipose tissue of obese mice such as KKAy mice and HFD-induced obese mice, reactive oxygen species production was accompanied by decreased expression of antioxidant enzymes ${ }^{(29)}$.

In the present study, another candidate gene for controlling energy expenditure is the bone morphogenetic protein 4 (Bmp4) gene. Recently, Qian et al. ${ }^{(30)}$ reported that adiposespecific overexpression of $B m p 4$ led to reduced body fat mass and increased energy expenditure, whereas Bmp4deficient mice exhibited increased adiposity and impaired insulin sensitivity, which suggests that $B m p 4$ can regulate WAT remodelling and induction of brown adipocyte-like cell structure and function. Interestingly, along with $B m p 4$, multiple genes involved in skeletal system development and pattern specification were down-regulated in the present study. Similarly, down-regulation of homeobox genes, including Hoxa5, was observed in the epididymal WAT of ob/ob mice compared with lean controls ${ }^{(31)}$, whereas expression of homeobox genes (e.g. Hoxa5, Hoxb5, Hoxc6 and Irx3) was up-regulated after fat loss in human subjects ${ }^{(32)}$. Recently, Gehrke et al. ${ }^{(33)}$ suggested epigenetic regulation, in particular DNA methylation of $H O X$ genes as a mechanism that explains
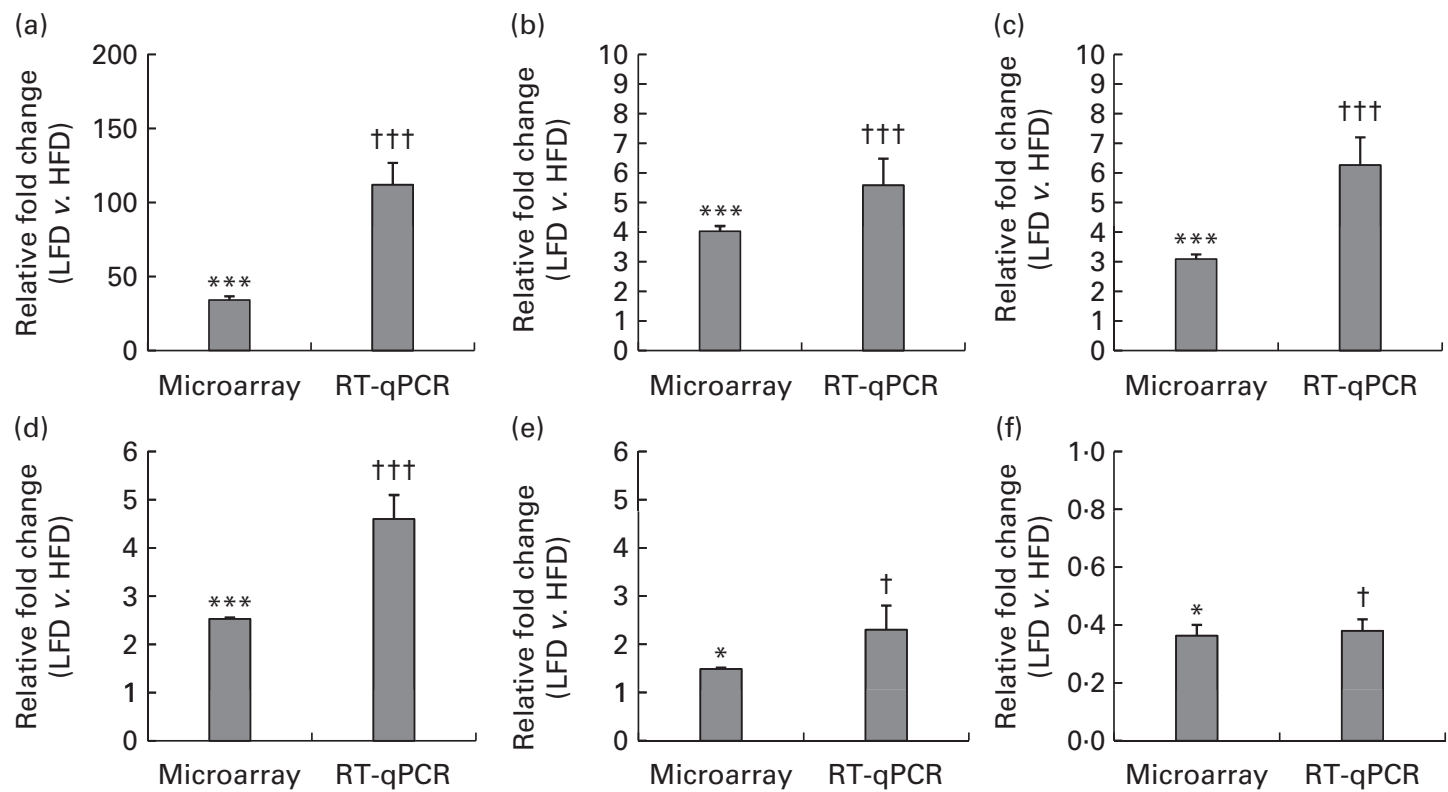

Fig. 2. Validation of microarray data using real-time quantitative PCR (RT-qPCR) for (a) Rgs1, (b) Mmp2, (c) Ccl2, (d) TIr2, (e) T/r4 and (f) Irs2. Values are means with their standard errors represented by vertical bars. ${ }^{*} P<0.05$, ${ }^{* * *} P<0.001$ (Wilcoxon $t$ test). $+P<0.05$, t† $P<0.001$ (Student's $t$ test). LFD, low-fat diet; HFD, high-fat diet. For definition of gene abbreviations, see online Supplementary Table S1. 
how these transcriptional factors exhibit such a distinct depotspecific expression pattern. Different adipose depots are known to mediate different effects on the risk for metabolic disorders ${ }^{(33)}$. Moreover, obese patients with type 2 diabetes had lower expression of $\operatorname{Ig} f 2$ in the adipose tissue compared with those who were lean ${ }^{(34)}$, and down-regulation of $\operatorname{Ig} f 2$ in the adipose tissue contributed to the paternal transmission of HFD-induced obesity ${ }^{(35)}$. However, many genes related to skeletal system development and pattern specification still have unknown roles in the development of obesity. Thus, understanding these genes will facilitate the development of novel approaches in the treatment of obesity.

In contrast, since the NEFA released by lipolysis or taken up by cells can be utilised in WAT as a source of energy through $\beta$-oxidation in the mitochondria, a decrease in the expression of genes involved in lipolysis and fatty acid uptake and transport in response to HFD may reduce $\beta$-oxidation, resulting in excessive fat accumulation. In mice, Adrb3 disruption reduces lipolysis and increases body fat accumulation ${ }^{(36)}$, and Adrb3 increases thermogenesis in the brown adipose tissue $^{(37)}$. Deficiency of Fabp4 also reduces lipolysis in mice $^{(38)}$, and a decrease in Fabp4 expression in the adipose tissue has been found to be inversely associated with obesity in human subjects ${ }^{(39)}$

The expression of several GPCR genes, including Gpr64, Gpr120, Gpr109a, Gpr156 and Opn3, was down-regulated by HFD in the present study. Mice lacking Gpr120, an NEFA-sensing GPCR, exhibited obesity, glucose intolerance and fatty liver ${ }^{(40)}$. Furthermore, Gpr109a has been reported to act as a metabolic sensor activated by intermediates of energy metabolism, and its expression was down-regulated in the adipose tissue of HFD-fed mice ${ }^{(41)}$. In contrast, the expression of genes encoding RGS (Rgs1 and Rgs10) was up-regulated by HFD. The RGS family is involved in the rapid turn-off of GPCR signalling pathways ${ }^{(42)}$. Huang et al. ${ }^{(43)}$ demonstrated that RGS-insensitive Gai2G184S mice exhibited resistance to the metabolic effects of HFD, and were protected from insulin resistance by increasing energy expenditure and peripheral insulin sensitivity. Furthermore, in another study, Rgs2- and Rgs5-deficient mice had less body mass and body fat than littermate controls ${ }^{(44,45)}$. However, the specific roles of most GPCR and RGS genes in the adipose tissue are not yet fully understood.

Obesity is characterised by extensive reorganisation of the adipose tissue, which involves ECM remodelling and adipogenesis $^{(46)}$. Cathepsins, ADAM, MMP and their inhibitors (i.e. TIMP) are involved in ECM remodelling ${ }^{(47)}$. The present study indicated that mRNA levels of most genes involved in ECM remodelling were up-regulated in obese adipose tissues compared with lean tissues, while expression of Adam 7 and Mmp9 was down-regulated in obesity. Interestingly, mice lacking Ctsk, Ctsl, Adam12, Timp1 or Mmp2, but not Mmp9, were protected against obesity ${ }^{(48-53)}$. Belo et al. ${ }^{(54)}$ reported the association between $M m p 9$ gene polymorphisms and lower plasma Mmp9 level in obese children and adolescents, suggesting a potential role of $M m p 9$ gene in the development of obesity. We also observed that HFD up-regulated the expression of $\mathrm{Tg} f b 1$ (encoding a profibrotic cytokine), as well as genes encoding various ECM components, including members of the collagen family, and increased adipose collagen accumulation, suggesting that HFD may facilitate abnormal ECM accumulation in WAT, promoting fibrosis. In addition, we detected significant expression of genes for a lymphocyte chemotactic factor (CXcl12) and its receptor ( $C x$ r 4 4), as well as genes related to macrophage infiltration ( Ccl2, Cxcl14 and Ccr5) in WAT of HFD-fed mice. It is well known that macrophage infiltration plays a critical role in adipose tissue inflammation, and that chemokines are involved in this process. In obese mice, a deficiency of $\mathrm{Ccl} 2$, Cxcl14 or Ccr 5 decreased macrophage infiltration in the adipose tissue and improved metabolic function, while overexpression of $\mathrm{Ccl} 2$ in the adipose tissue caused the opposite phenotype ${ }^{(55-58)}$. Besides macrophages, T and B lymphocytes have been shown to accumulate in the adipose tissue of obese mice and have been associated with inflammation and insulin resistance ${ }^{(59,60)}$

Many other genes involved in innate or adaptive immune and inflammatory responses, such as those encoding toll-like receptors, C-type lectin receptors, Fc receptors, cytokine receptors and triggering receptors, were up-regulated by HFD. One of these genes, $\operatorname{Tl} r 2$, has been associated with increased adiposity, macrophage infiltration and inflammatory cytokine expression $^{(61)}$. Saa3, which encodes an inflammatory adipocytokine and may be a potential link between obesity and its metabolic complications ${ }^{(62)}$, was also one of the top ten genes up-regulated by HFD in the present study. However, despite the increased expression of multiple inflammatory genes in WAT of HFD-fed mice, plasma concentrations of inflammatory chemokines and cytokines were not affected, as described in a previous study ${ }^{(63)}$. Thomas et al. ${ }^{(63)}$ suggested that shifts in these inflammation markers in the blood are probably not as sensitive as WAT mRNA patterns, which reflect the inflammatory status of the local tissue.

Tph2, Ubd and Atf3 were also among the genes most highly up-regulated by HFD in the present study. Although a deficiency of $T p h 2$ or $U b d$ in mice caused body fat reduction by increasing energy expenditure ${ }^{(64,65)}$, the specific functions of these genes in the adipose tissue are still unclear. Atf3 has been reported to act as a negative regulator of SFA/TLR 4 signalling and macrophage activation in obese adipose tissue $^{(66)}$; however, overexpression of Atf3 decreased mitochondrial function and mitochondria-related gene expression in adipocytes in vivo and in vitro ${ }^{(67)}$. Thus, further experiments are required to evaluate whether Atf3 has a beneficial or detrimental role in obesity and its associated metabolic disturbances.

In conclusion, long-term HFD feeding predominantly up-regulated a series of genes involved in immune and inflammatory responses, as well as cell activation and division in WAT of C57BL/6J mice. Genes down-regulated by HFD were mainly implicated in fatty acid metabolism, oxidationreduction, insulin response and skeletal system development. The top ten up-regulated genes were Rgs1, Mmp12, Gpnmb, Trem2, Tph2, Saa3, Ubd, Atf3, Itgad and Cd68, which might be associated with energy expenditure (Rgs1, Tph2, Ubd and Atf3), ECM remodelling and components (Mmp12, Gpnmb 
and Itgad) and inflammation (Trem2, Saa3, Atf3 and Cd68); further studies are needed to evaluate the roles of these genes in the pathophysiology of the adipose tissue. The functions of the top ten down-regulated genes, except Pck and Fabp 4, are also poorly understood. Thus, the present findings will be useful in future efforts to identify the therapeutic targets for obesity. However, further studies are needed to investigate the molecular mechanisms responsible for WAT depot-specific differences.

\section{Supplementary material}

To view supplementary material for this article, please visit http://dx.doi.org/10.1017/S0007114515000100

\section{Acknowledgements}

The present study was supported by the Basic Science

Research Program (U. J. J., grant no. NRF-2011-0022387, NRF-2014R1A1A4A01007858), the SRC program (M.-S. C., Center for Food \& Nutritional Genomics, grant no. 20080062618) and the fundamental technology program (M.-S. C., grant no. 2012M3A9C4048818) of the National Research Foundation of Korea funded by the Ministry of Education, Science and Technology. This study received no specific grant from any funding agency, commercial or not-for-profit sectors. In addition, all the funders had no role in the design and analysis of the study or in the writing of this article.

The authors' contributions are as follows:

M.-S. C. designed the study and participated in the writing of the manuscript; Y.-J. K. performed the animal experiments; J. Y. R. and S. R K. were involved in data interpretation and edited the manuscript; U. J. J. analysed the data and wrote the manuscript. All authors read and approved the final manuscript.

The authors declare that there is no conflict of interest.

\section{References}

1. Jung UJ \& Choi MS (2014) Obesity and its metabolic complications: the role of adipokines and the relationship between obesity, inflammation, insulin resistance, dyslipidemia and nonalcoholic fatty liver disease. Int J Mol Sci 15, 6184-6223.

2. Rolls BJ \& Hammer VA (1995) Fat, carbohydrate, and the regulation of energy intake. Am J Clin Nutr 62, 1086S-1095S.

3. Savastano DM \& Covasa M (2005) Adaptation to a high-fat diet leads to hyperphagia and diminished sensitivity to cholecystokinin in rats. J Nutr 135, 1953-1959.

4. Buettner R, Newgard CB, Rhodes CJ, et al. (2000) Correction of diet-induced hyperglycemia, hyperinsulinemia, and skeletal muscle insulin resistance by moderate hyperleptinemia. Am J Physiol Endocrinol Metab 278, E563-E569.

5. Leibowitz SF, Dourmashkin JT, Chang GQ, et al. (2004) Acute high-fat diet paradigms link galanin to triglycerides and their transport and metabolism in muscle. Brain Res 1008, $168-178$.

6. Do GM, Oh HY, Kwon EY, et al. (2011) Long-term adaptation of global transcription and metabolism in the liver of high-fat diet-fed C57BL/6J mice. Mol Nutr Food Res 55 S173-S185.

7. Kalupahana NS, Voy BH, Saxton AM, et al. (2011) Energyrestricted high-fat diets only partially improve markers of systemic and adipose tissue inflammation. Obesity (Silver Spring) 19, 245-254.

8. Oscai LB, Miller WC \& Arnall DA (1987) Effects of dietary sugar and of dietary fat on food intake and body fat content in rats. Growth 51, 64-73.

9. Hulman S \& Falkner B (1994) The effect of excess dietary sucrose on growth, blood pressure, and metabolism in developing Sprague-Dawley rats. Pediatr Res 36, 95-101.

10. Santos FL, Esteves SS, da Costa Pereira A, et al. (2012) Systematic review and meta-analysis of clinical trials of the effects of low carbohydrate diets on cardiovascular risk factors. Obes Rev 13, 1048-1066.

11. Ruth MR, Port AM, Shah M, et al. (2013) Consuming a hypocaloric high fat low carbohydrate diet for 12 weeks lowers C-reactive protein, and raises serum adiponectin and high density lipoprotein-cholesterol in obese subjects. Metabolism 62, 1779-1787.

12. Hageman RS, Wagener A, Hantschel C, et al. (2010) High-fat diet leads to tissue-specific changes reflecting risk factors for diseases in DBA/2J mice. Physiol Genomics 42, 55-66.

13. Bjørndal B, Burri L, Staalesen V, et al. (2011) Different adipose depots: their role in the development of metabolic syndrome and mitochondrial response to hypolipidemic agents. J Obes 2011, 490650.

14. Chau YY, Bandiera R, Serrels A, et al. (2014) Visceral and subcutaneous fat have different origins and evidence supports a mesothelial source. Nat Cell Biol 16, 367-375.

15. Briones AM, Nguyen Dinh Cat A, Callera GE, et al. (2012) Adipocytes produce aldosterone through calcineurindependent signaling pathways: implications in diabetes mellitus-associated obesity and vascular dysfunction. Hypertension 59, 1069-1078.

16. Xu H, Barnes GT, Yang Q, et al. (2003) Chronic inflammation in fat plays a crucial role in the development of obesityrelated insulin resistance. J Clin Invest 112, 1821-1830.

17. Altintas MM, Azad A, Nayer B, et al. (2011) Mast cells, macrophages, and crown-like structures distinguish subcutaneous from visceral fat in mice. J Lipid Res 52, 480-488.

18. Liu LF, Shen WJ, Ueno M, et al. (2013) Age-related modulation of the effects of obesity on gene expression profiles of mouse bone marrow and epididymal adipocytes. PLOS ONE 8, e72367.

19. Do GM, Kwon EY, Kim E, et al. (2010) Hepatic transcription response to high-fat treatment in mice: microarray comparison of individual vs. pooled RNA samples. Biotechnol J 5, 970-973.

20. Lin S, Thomas TC, Storlien LH, et al. (2000) Development of high fat diet-induced obesity and leptin resistance in C57Bl/ 6J mice. Int J Obes Relat Metab Disord 24, 639-646.

21. Flachs P, Rossmeisl M, Kuda O, et al. (2013) Stimulation of mitochondrial oxidative capacity in white fat independent of UCP1: a key to lean phenotype. Biochim Biophys Acta 1831, 986-1003.

22. Valerio A, Cardile A, Cozzi V, et al. (2006) TNF-alpha downregulates eNOS expression and mitochondrial biogenesis in fat and muscle of obese rodents. J Clin Invest 116, 2791-2798.

23. Böttcher H \& Fürst P (1997) Decreased white fat cell thermogenesis in obese individuals. Int J Obes Relat Metab Disord 21, 439-444. 
24. Kusminski CM \& Scherer PE (2012) Mitochondrial dysfunction in white adipose tissue. Trends Endocrinol Metab 23 435-443.

25. Blüher M, Engeli S, Klöting N, et al. (2006) Dysregulation of the peripheral and adipose tissue endocannabinoid system in human abdominal obesity. Diabetes 55, 3053-3060.

26. Vaitheesvaran B, Yang L, Hartil K, et al. (2012) Peripheral effects of FAAH deficiency on fuel and energy homeostasis: role of dysregulated lysine acetylation. PLOS ONE 7, e33717.

27. Muoio DM \& Neufer PD (2012) Lipid-induced mitochondrial stress and insulin action in muscle. Cell Metab 15, 595-605.

28. Tormos KV, Anso E, Hamanaka RB, et al. (2011) Mitochondrial complex III ROS regulate adipocyte differentiation. Cell Metab 14, 537-544.

29. Furukawa S, Fujita T, Shimabukuro M, et al. (2004) Increased oxidative stress in obesity and its impact on metabolic syndrome. J Clin Invest 114, 1752-1761.

30. Qian SW, Tang Y, Li X, et al. (2013) BMP4-mediated brown fat-like changes in white adipose tissue alter glucose and energy homeostasis. Proc Natl Acad Sci U S A 110, E798-E807.

31. Yamamoto Y, Gesta S, Lee KY, et al. (2010) Adipose depots possess unique developmental gene signatures. Obesity (Silver Spring) 18, 872-878.

32. Dankel SN, Fadnes DJ, Stavrum AK, et al. (2010) Switch from stress response to homeobox transcription factors in adipose tissue after profound fat loss. PLOS ONE 5, e11033.

33. Gehrke S, Brueckner B, Schepky A, et al. (2013) Epigenetic regulation of depot-specific gene expression in adipose tissue. PLOS ONE 8, e82516.

34. Chen M, Macpherson A, Owens J, et al. (2012) Obesity alone or with type 2 diabetes is associated with tissue specific alterations in DNA methylation and gene expression of PPARGC1A and IGF2. J Diabetes Res Clin Metab 1, 1-8.

35. Morita S, Horii T, Kimura M, et al. (2014) Paternal allele influences high fat diet-induced obesity. PLOS ONE 9, e85477.

36. Revelli JP, Preitner F, Samec S, et al. (1997) Targeted gene disruption reveals a leptin-independent role for the mouse beta3-adrenoceptor in the regulation of body composition. J Clin Invest 100, 1098-1106.

37. Arner P \& Hoffstedt J (1999) Adrenoceptor genes in human obesity. J Intern Med 245, 667-672.

38. Scheja L, Makowski L, Uysal KT, et al. (1999) Altered insulin secretion associated with reduced lipolytic efficiency in aP2 ${ }^{-1-}$ mice. Diabetes 48, 1987-1994.

39. Queipo-Ortuño MI, Escoté X, Ceperuelo-Mallafré V, et al. (2012) FABP 4 dynamics in obesity: discrepancies in adipose tissue and liver expression regarding circulating plasma levels. PLOS ONE 7, e 48605.

40. Ichimura A, Hirasawa A, Poulain-Godefroy O, et al. (2012) Dysfunction of lipid sensor GPR120 leads to obesity in both mouse and human. Nature $\mathbf{4 8 3}, 350-354$.

41. Wanders D, Graff EC \& Judd RL (2012) Effects of high fat diet on GPR109A and GPR81 gene expression. Biochem Biophys Res Commun 425, 278-283.

42. De Vries L, Zheng B, Fischer T, et al. (2000) The regulator of G protein signaling family. Annu Rev Pharmacol Toxicol 40 $235-271$

43. Huang X, Charbeneau RA, Fu Y, et al. (2008) Resistance to diet-induced obesity and improved insulin sensitivity in mice with a regulator of $G$ protein signaling-insensitive G184S Gnai2 allele. Diabetes 57, 77-85.

44. Cho H, Park C, Hwang IY, et al. (2008) Rgs5 targeting leads to chronic low blood pressure and a lean body habitus Mol Cell Biol 28, 2590-2597.
45. Nunn C, Zhao P, Zou MX, et al. (2011) Resistance to agerelated, normal body weight gain in RGS2 deficient mice. Cell Signal 23, 1375-1386.

46. Crandall DL, Hausman GJ \& Kral JG (1997) A review of the microcirculation of adipose tissue: anatomic, metabolic, and angiogenic perspectives. Microcirculation 4, 211-232.

47. Järveläinen H, Sainio A, Koulu M, et al. (2009) Extracellular matrix molecules: potential targets in pharmacotherapy. Pharmacol Rev 61, 198-223.

48. Lijnen HR, Demeulemeester D, Van Hoef B, et al. (2003) Deficiency of tissue inhibitor of matrix metalloproteinase-1 (TIMP-1) impairs nutritionally induced obesity in mice. Thromb Haemost 89, 249-255.

49. Masaki M, Kurisaki T, Shirakawa K, et al. (2005) Role of meltrin \{alpha\} (ADAM12) in obesity induced by high-fat diet. Endocrinology 146, 1752-1763.

50. Van Hul M \& Lijnen HR (2008) A functional role of gelatinase $\mathrm{A}$ in the development of nutritionally induced obesity in mice. J Thromb Haemost 6, 1198-1206.

51. Yang M, Zhang Y, Pan J, et al. (2007) Cathepsin L activity controls adipogenesis and glucose tolerance. Nat Cell Biol 9, 970-977.

52. Yang M, Sun J, Zhang T, et al. (2008) Deficiency and inhibition of cathepsin $\mathrm{K}$ reduce body weight gain and increase glucose metabolism in mice. Arterioscler Thromb Vasc Biol 28, 2202-2208.

53. Van Hul M, Piccard H \& Lijnen HR (2010) Gelatinase B (MMP-9) deficiency does not affect murine adipose tissue development. Thromb Haemost 104, 165-171.

54. Belo VA, Souza-Costa DC, Luizon MR, et al. (2012) Matrix metalloproteinase-9 genetic variations affect MMP-9 levels in obese children. Int J Obes (Lond) 36, 69-75.

55. Kanda H, Tateya S, Tamori Y, et al. (2006) MCP-1 contributes to macrophage infiltration into adipose tissue, insulin resistance, and hepatic steatosis in obesity. J Clin Invest 116, $1494-1505$.

56. Kamei N, Tobe K, Suzuki R, et al. (2006) Overexpression of monocyte chemoattractant protein-1 in adipose tissues causes macrophage recruitment and insulin resistance. J Biol Chem 281, 26602-26614

57. Nara N, Nakayama Y, Okamoto S, et al. (2007) Disruption of CXC motif chemokine ligand-14 in mice ameliorates obesityinduced insulin resistance. J Biol Chem 282, 30794-30803.

58. Kitade H, Sawamoto K, Nagashimada M, et al. (2012) CCR5 plays a critical role in obesity-induced adipose tissue inflammation and insulin resistance by regulating both macrophage recruitment and M1/M2 status. Diabetes 61, 1680-1690

59. Kintscher U, Hartge M, Hess K, et al. (2008) T-lymphocyte infiltration in visceral adipose tissue: a primary event in adipose tissue inflammation and the development of obesity-mediated insulin resistance. Arterioscler Thromb Vasc Biol 28, 1304-1310.

60. Winer DA, Winer S, Shen L, et al. (2011) B cells promote insulin resistance through modulation of $\mathrm{T}$ cells and production of pathogenic IgG antibodies. Nat Med 17, 610-617.

61. Himes RW \& Smith CW (2010) Tlr2 is critical for diet-induced metabolic syndrome in a murine model. FASEB $I \mathbf{2 4}$ 731-739.

62. Yang RZ, Lee MJ, Hu H, et al. (2006) Acute-phase serum amyloid A: an inflammatory adipokine and potential link between obesity and its metabolic complications. PLoS Med 3, 884-894.

63. Thomas AP, Dunn TN, Oort PJ, et al. (2011) Inflammatory phenotyping identifies CD11d as a gene markedly induced 
in white adipose tissue in obese rodents and women. $J$ Nutr 141, 1172-1180

64. Yadav VK, Oury F, Suda N, et al. (2009) A serotonin-dependent mechanism explains the leptin regulation of bone mass, appetite, and energy expenditure. Cell 138, 976-989.

65. Canaan A, Defuria J, Perelman E, et al. (2014) Extended lifespan and reduced adiposity in mice lacking the FAT1O gene. Proc Natl Acad Sci U S A 111, 5313-5318.
66. Suganami T, Yuan X, Shimoda Y, et al. (2009) Activating transcription factor 3 constitutes a negative feedback mechanism that attenuates saturated fatty acid/toll-like receptor 4 signaling and macrophage activation in obese adipose tissue. Circ Res 105, 25-32.

67. Jang MK, Son Y \& Jung MH (2013) ATF3 plays a role in adipocyte hypoxia-mediated mitochondria dysfunction in obesity. Biochem Biophys Res Commun 431, 421-427. 